\title{
Die SPD im Spagat zwischen Regierungsverantwortung und Überzeugung - Migration im parlamentarischen Parteienwettbewerb
}

\author{
Dana Siobhan Atzpodien (1)
}

Online publiziert: 19. Mai 2020

(C) Der/die Autor(en) 2020

Zusammenfassung Der vorliegende Beitrag analysiert die flüchtlings- und migrationspolitischen Reden des Deutschen Bundestags während der 18. Legislaturperiode und zeigt mithilfe des quantitativen Textanalyseinstruments Wordfish sowie einer umfangreichen qualitativen Validierung die Dynamiken des parlamentarischen Parteienwettbewerbs während der europäischen Flüchtlingskrise. Das dynamische Feld der Flüchtlings- und Migrationspolitik stellt die Parteien und insbesondere die Sozialdemokratische Partei Deutschlands (SPD) vor dem Hintergrund des liberalen Dilemmas sowie durch wechselnde Themenbetonung und daraus folgende Wettbewerbslogiken unter Druck. Die Position der SPD variierte im sonst stabilen parlamentarischen Wettbewerb deutlich, da sich die Partei in einem Spagat zwischen ihrer formalen Regierungsverantwortung und ihrer ideologischen Positionierung befand.

Schlüsselwörter Bundestag · SPD · Flüchtlingskrise · Parteienwettbewerb · Parlament $\cdot$ Quantitative Textanalyse

Zusatzmaterial online Zusätzliche Informationen sind in der Online-Version dieses Artikels (https:// doi.org/10.1007/s12286-020-00445-6) enthalten.

D. S. Atzpodien $(\bowtie)$

Institut für Politikwissenschaft, Westfälische Wilhelms-Universität Münster,

Scharnhorststraße 100, 48151 Münster, Deutschland

E-Mail: atzpodien@uni-muenster.de 


\title{
The SPD in a balancing act between government responsibility and conviction-Migration in parliamentary party competition
}

\begin{abstract}
This paper analyses refugee and migration policy speeches of the German Bundestag during the 18th legislative period and shows the dynamics of parliamentary party competition during the European refugee crisis using the quantitative text analysis tool Wordfish and a comprehensive qualitative validation. The dynamic field of refugee and migration policy puts parties and in particular the Social Democratic Party of Germany (SPD) under pressure against the background of the "liberal dilemma" as well as through changing thematic emphasis and the resulting logic of competition. The position of the SPD varied considerably in the otherwise stable parliamentary competition as the party found itself in a balancing act between its formal government responsibility and its ideological position.
\end{abstract}

Keywords Bundestag - SPD · Refugee crisis · Party competition · Parliament · Quantitative text analysis

\section{Einleitung}

Während der 18. Legislaturperiode des Deutschen Bundestags und der Zeit der sogenannten Flüchtlingskrise $e^{1}$ kommt es im Sommer 2015 zum bisherigen Höhepunkt der Flüchtlingszuwanderung nach Europa. Sie wird zum zentralen Thema des Parteienwettbewerbs in Deutschland (Dostal 2017b; Kortmann und Stecker 2017; Wiesendahl 2016). Obwohl sich Deutschland schon langsam als Einwanderungsland versteht, setzen die Entwicklungen während der Flüchtlingskrise nicht nur die Gesellschaft, sondern auch die Parteien unter Druck. Die 2014 deutlich ansteigenden Flüchtlingszahlen führten zu einer Überforderung der Behörden sowie einer Überpräsenz des Themas in den Medien, die zu einem polarisierten öffentlichen und politischen Diskurs führten (Haller 2017). Die Rufe nach schnellen politischen Lösungen und Positionierungen der Parteien wurden immer lauter. Der hohe Problemdruck destabilisierte die Regierungsfraktionen auch für die Öffentlichkeit sichtbar. So wurde der Kurs der Kanzlerin nicht einheitlich unterstützt und auch die Koalition der SPD mit der CDU/CSU-Fraktion war von grundlegenden Meinungsverschiedenheiten bestimmt (Spiegel Online 2015b; Süddeutsche Zeitung 2016b).

Es stellt sich daher die Frage, wie sich die Parteien während der Legislaturperiode im Politikfeld Flucht und Migration positionieren und agieren. Dies ist in dem genannten Politikfeld von besonderem Interesse, da es sich konzeptionell um ein facettenreiches Feld handelt, das auch in der parlamentarischen Debatte durch die Bearbeitung und Betonung verschiedener Aspekte unterschiedliche Wettbewerbsdynamiken und Positionierungen für die Parteien zulässt (Hollifield 2015; Lutz 2019). Der vorliegende Beitrag nimmt an, dass sich die Parteien im Bundestag durch die

\footnotetext{
1 Der Begriff Flüchtlingskrise wird in diesem Artikel in Anlehnung an die Medienberichterstattung genutzt. Die mitschwingende Wertung der steigenden Asylantragzahlen als Krise in der Legislaturperiode des 18. Deutschen Bundestags von Ende 2013 bis 2017 wird jedoch nicht geteilt.
} 
Überlappung verschiedener Dimensionen im untersuchten Politikfeld entsprechend der allgemeinen Links-Rechts-Dimension positionieren (Polk et al. 2017), und argumentiert, dass unter anderem die Mehrdimensionalität des Politikfeldes unterschiedliche Themenbetonungen in der parlamentarischen Debatte nach sich zog, die andere Wettbewerbslogiken für die Parteien ermöglichten. Dies zeigt sich besonders an der ambivalenten Position der SPD. Die SPD nahm während der Flüchtlingskrise eine widersprüchliche Position im Bundestag ein und stand in einem Spannungsverhältnis zwischen ihrer Regierungszugehörigkeit und ideologischen Positionierung. Neben der ideologischen Ausrichtung sowie den Dynamiken des Politikfeldes hat auch die institutionelle Logik der parlamentarischen Arena ganz wesentlichen Einfluss auf die Positionierung der Parteien. Dabei spielt die Zugehörigkeit der Parteien zur Opposition oder Regierungskoalition eine entscheidende Rolle. Dies zeigt sich ebenso an der prekären Position der SPD, die sich auf der Links-Rechts-Dimension ideologisch dichter an den Oppositionsparteien befindet als an den anderen Regierungsparteien (ebd.).

Empirisch gezeigt wird dies anhand einer quantitativen Textanalyse aller Bundestagsdebatten der 18. Legislaturperiode (2013-2017) zur Flüchtlings- und Migrationsthematik sowie einer qualitativ strukturierten Inhaltsanalyse. Im ersten Schritt ist es mit dem Textanalyseinstrument Wordfish möglich, die Parlamentsreden in fünf Analysezeiträumen zur Flüchtlings- und Migrationspolitik zu skalieren und Parteipositionen zu bestimmen. Die aus den Reden gewonnenen Parteienpositionen sind neben den Einschätzungen von Expertinnen und Experten sowie durch die Bestimmung von Wahlprogrammanalysen wertvolle Informationsquellen. Wordfish wurde für die Analyse von Parlamentsdebatten entwickelt und für mehrere Sprachen und Parlamentsarten sowie Lobbying-Statements, Abgeordnetenbiographien und auch für Wahlprogramme erfolgreich angewandt (Aydogan und Slapin 2015; Catalinac 2018; Frid-Nielsen 2018; Klüver 2012; Lo et al. 2016; Marcinkiewicz und Tepe 2012). Im zweiten Schritt ermöglicht die qualitative Analyse einen tieferen Blick in die Argumentationsstruktur der Parteien sowie in die Wettbewerbsdynamik, indem je eine thematisch repräsentative Debatte pro Analysezeitraum untersucht wird.

Der Artikel ist wie folgt strukturiert: Der nächste Abschnitt stellt die Erkenntnisse der bestehenden Forschung zur Migrationspolitik in Deutschland vor und erarbeitet die Herausforderungen im vielfältigen Politikfeld Migration, insbesondere für Regierungsparteien in liberalen Staaten wie Deutschland. Mit der Zusammenführung dieser beiden Gebiete wird zunächst eine Hypothese zur Reihung der Parteien in Deutschland während der Flüchtlingskrise, dann eine Erwartung zur parlamentarischen Dynamik und schließlich die besondere Herausforderung der SPD in dieser Konstellation zur Zeit der Flüchtlingskrise konzeptualisiert. Nach der Präsentation der Daten und Methoden folgt die empirische Analyse. Die abschließende Diskussion fasst die Ergebnisse zusammen und diskutiert sie vor dem Hintergrund der vorher formulierten theoretischen Erwartungen. 


\section{Forschungsstand und Hypothesen}

\subsection{Migrationspolitik in Deutschland}

Um die Dynamiken des Parteiensystems während der Flüchtlingskrise im Politikfeld Migration bedeutungsvoll analysieren zu können, ist zunächst zu definieren, welche Dimensionen das Politikfeld umfasst und welche deutschen Debatten in ihm verortet werden können. Dies ist besonders wichtig, da es sich um ein dynamisches und facettenreiches Politikfeld handelt, bei dem eine einheitliche Abgrenzung zu anderen Politikbereichen schwerfällt (Gestmann und Hilz 2017). In der vorliegenden Arbeit ist sowohl Flüchtlings- und Migrationspolitik als auch Integrationspolitik gemeint. Diese unterschiedlichen Facetten bieten Raum für variierende Positionierungen und Wettbewerbsdynamiken der Parteien (Lutz 2019; Money 1999). In den meisten europäischen Ländern basiert Migrationspolitik auf einem parteilichen Konsens, der einer wirtschaftlichen Logik folgt, während die Integrationspolitik einer parteiischen Logik folgt, die durch den Wahlkampf bestimmt wird (Givens und Luedtke 2005). Beim parteiischen Konsens wird auch von einem „Gentlemen-Agreement“ der Parteien gesprochen (Kortmann und Stecker 2017), da alle Parteien sich einen Wettbewerbsvorteil versprechen, wenn das Thema nicht auf die Agenda gesetzt wird.

Eine forcierte Trennung der Facetten als eigenständige Politikfelder ergibt wenig Sinn, da dies nicht die Interdependenzen im parteipolitischen Wettbewerb in Deutschland widerspiegelt, weder historisch, noch gegenwärtig (Borkert und Bosswick 2011; Gestmann und Hilz 2017). Es ist zu bezweifeln, dass die Verhandlungen in den politischen Bereichen während der Flüchtlingskrise sowohl von den Parteien als auch von der Wählerschaft so getrennt werden können; daher werden sie in der vorliegenden Analyse als ein Politikfeld betrachtet. Aus dieser Konzeption ist zugleich abzuleiten, dass das Politikfeld über die Zeit nicht konsistent sein kann und dass durch unterschiedliche thematische Betonungen variierende Wettbewerbsbedingungen und Positionierungen der Parteien hervorgerufen werden können. Des Weiteren treffen in dem Politikfeld widersprüchliche Interessen aufeinander, wie beispielsweise der Bedarf nach Arbeitsmarktmigration und Forderungen nach restriktiveren Migrationspolitiken (Boswell 2009b; Rosenblum und Cornelius 2012). Hollifield (2015) spricht von einem liberalen Dilemma, da liberale Staaten einerseits auf Offenheit setzen, die sie im Politikfeld Migration auch Flüchtlingen und Migranten gewähren wollen. Andererseits steht die liberale Handlungsmaxime aber potentiell im Gegensatz zum Ziel, der einheimischen Bevölkerung möglichst gute wirtschaftliche Bedingungen zu gewährleisten oder den Zugang zu Sozialsystemen zu regulieren.

Das Politikfeld Migration hat in Deutschland bereits einen starken Wandel erlebt (Bieber et al. 2017; Blätte 2015; Heckmann und Wiest 2015; Hess und Green 2016). Vor der Flüchtlingskrise wird Migration von der Politik und den Parteien überwiegend aus einem Blickwinkel der Arbeitsmarktmigration bearbeitet, d.h. aus wirtschaftspolitischer Sicht (Niedermayer 2018), Integration hingegen mit dem Ziel, ,....Menschen mit Migrationshintergrund zu ermöglichen, chancengleich und gleichberechtigt am sozialen, politischen, kulturellen und wirtschaftlichen Leben teilzunehmen..." (Gestmann und Hilz 2017, S. 220). Dabei folgen die Parteien einer 
Tab. 1 Entwicklung der deutschen Flüchtlings- und Migrationspolitik seit 1955. (Quelle: Übersetzung von Heckmann und Wiest 2015; mit eigenen Ergänzungen von 2012-2018)

\begin{tabular}{|c|c|}
\hline $\begin{array}{l}\text { Definition der Einwande- } \\
\text { rungssituation }\end{array}$ & Entwicklung der Flüchtlings- und Migrationspolitik \\
\hline $\begin{array}{l}1955-1973 \\
\text { Einstellung von } \\
\text { Leiharbeitnehmern }\end{array}$ & $\begin{array}{l}\text { Keine spezifische Integrationspolitik } \\
\text { Eingliederung in Sozialstaatseinrichtungen }\end{array}$ \\
\hline $\begin{array}{l}\text { 1973-1989/1990 } \\
\text { Verweigerung der } \\
\text { Einwanderungssituation }\end{array}$ & $\begin{array}{l}\text { Kontroversen über Integrationspolitik } \\
\text { Ernennung eines „Beauftragten für Ausländer“ } \\
\text { Unterstützung der freiwilligen Rückkehr von Arbeitsmigrantinnen und } \\
\text { Arbeitsmigranten }\end{array}$ \\
\hline $\begin{array}{l}\text { 1990-1998 } \\
\text { Neue Einwanderung und } \\
\text { Einleitung } \\
\text { eines politischen } \\
\text { Paradigmenwechsels }\end{array}$ & $\begin{array}{l}\text { Anhaltende Verweigerung der Einwanderungssituation und Fehlen um- } \\
\text { fassender politischer Konzepte auf nationaler Ebene } \\
1998 \text { Regierungswechsel und offizielle An- } \\
\text { erkennung der Einwanderungssituation }\end{array}$ \\
\hline $\begin{array}{l}2000-2013 \\
\text { Neue Integrationspolitik }\end{array}$ & $\begin{array}{l}2000 \text { Neues Staatsbürgerschaftsgesetz } \\
2005 \text { Neues Zuwanderungsgesetz } \\
2006 \text { Deutsche Islamkonferenz } \\
2007 \text { Nationaler Integrationsplan } \\
2008 \text { Asylgesetz } \\
2011 \text { Nationaler Aktionsplan } \\
2012 \text { Umsetzung der Hochqualifiziertenrichtlinie der Europäischen Uni- } \\
\text { on (Blue Card EU) }\end{array}$ \\
\hline $\begin{array}{l}\text { Seit } 2014 \\
\text { Aktuelle Flüchtlings- und } \\
\text { Migrationspolitik }\end{array}$ & $\begin{array}{l}2014 \text { Änderung des Staatsangehörigkeitsgesetzes } \\
2015 \text { Änderungen im Asylrecht: Asylverfahrensbeschleunigungsgesetz - } \\
\text { Asylpaket I } \\
2015 \text { Datenaustauschverbesserungsgesetz } \\
2016 \text { Integrationsgesetz } \\
2016 \text { Einführung beschleunigter Asylverfahren - Asylpaket II } \\
2017 \text { Zweites Gesetz zur Änderung des Asylgesetzes } \\
2018 \text { Drittes Gesetz zur Änderung des Asylgesetzes }\end{array}$ \\
\hline
\end{tabular}

parteiischen Logik, die durch den Wahlkampf bestimmt wird (Lutz 2019). Heckmann und Wiest (2015) gliedern in ihrer Betrachtung das Politikfeld Migrationsund Integrationspolitik von 1995-2011 in vier Phasen auf (Tab. 1). Für die vorliegende Analyse wird das Politikfeld um die Dimension Flüchtlingspolitik und eine fünfte Phase ergänzt, da im Zeitraum der Flüchtlingskrise vorwiegend diese Dimension von den Parteien bearbeitet wird. Es zeigt sich hier die Entwicklung von Migrations- und Integrationsdebatten hin zu einer Kombination mit flüchtlingspolitischen Themen. Daher wird im weiteren Verlauf auch vom Politikfeld Flüchtlingsund Migrationspolitik gesprochen.

\subsection{Parteienwettbewerb in Deutschland}

Die Analyse des Parteienwettbewerbs baut auf den breit rezipierten Konzepten des Wettbewerbs um Themen (Issue Competition) sowie dem Wettbewerb um politische 
Positionen (Spatial Competition) auf (Budge 1994; Petrocik 1996; Riker 1993). Dem Wettbewerb um Positionen ist der Themenwettbewerb vorgeschaltet, da die Parteien so versuchen von ihnen favorisierte Themen auf die politische Agenda zu setzen. Im Fokus der Analyse steht der Wettbewerb um politische Positionen, mit denen sich die Parteien im parlamentarischen Wettbewerb behaupten müssen, da das Thema Migration durch den hohen Problemdruck nicht von der Agenda zu nehmen ist.

Um die Bedeutung des Politikfelds in Deutschland verstehen zu können, ist eine Betrachtung der Salienz hilfreich. Die Themenkonjunktur der Flüchtlings- und Migrationspolitik vor der Flüchtlingskrise lässt sich parallel zur thematischen Aufarbeitung anhand der Asylantragszahlen abbilden (siehe Abb. 1). So findet das Thema immer wieder Einzug in den parteipolitischen Diskurs, vor allem wenn steigende Asylantragszahlen Druck auf die Parteien ausüben, politisch aktiv zu werden. Die Flüchtlings- und Migrationspolitik ist trotz ihrer zwischenzeitlich gestiegenen Salienz und immer wiederkehrender Aktualität dennoch kein Kernthema des deutschen Wahlkampfs bzw. des Parteienwettbewerbs im Allgemeinen (Green 2006). Die Parteien sind sich der Risiken der Bearbeitung dieses Politikfeldes bewusst und wählen die Strategie des „Gentlemen-Agreements“ bzw. des parteiischen Konsenses, der einer wirtschaftlichen Logik folgt, da es mit hohen elektoralen Kosten verbunden sein kann, sich zu positionieren (Givens und Luedtke 2005; Kortmann und Stecker 2017).

\subsection{Parlamentarischer Parteienwettbewerb: Ideologische Positionierung}

Die Auseinandersetzung mit der Flüchtlings- und Migrationspolitik westeuropäischer Staaten birgt durch die facettenreiche Gestaltung des Politikfeldes konzeptionelle Besonderheiten für die Parteienpositionierung. So lässt sich das Politikfeld in Deutschland keiner der beiden dominanten Konfliktlinien (wirtschafts- oder gesellschaftspolitisch) eindeutig zuordnen, da eine Vielzahl von unterschiedlichen Politikbereichen angeschnitten wird. Dies können je nach Framing der Parteien oder der Öffentlichkeit ökonomische Facetten, Themen der nationalen Geschlossenheit bzw. des nationalen Selbstverständnisses sowie rechtliche Fragen der Staatsangehörigkeit und emotional aufgeladene Fragestellungen wie Moralvorstellungen oder damit verbundene Traditionen sein (Boswell 2009a; Brettell und Hollifield 2008; Odmalm 2011). Darüber hinaus folgen die unterschiedlichen Themen den bereits erläuterten unterschiedlichen Wettbewerbslogiken: die Flüchtlings- und Migrationspolitik einem parteiischen Konsens, der aus einer wirtschaftlichen Logik resultiert, während die Integrationspolitik einer parteiischen Logik folgt, die durch den Wahlkampf bestimmt wird (Givens und Luedtke 2005).

Ein Literaturstrang sieht insbesondere in diesem Politikfeld die Herausbildung einer neuen gesellschaftlichen Konfliktlinie, anhand derer einerseits libertär-kosmopolitische und andererseits autoritär-nationalistische Positionen entstehen und die speziell als Folge der Globalisierung eintrete (Calhoun 2007; Kriesi et al. 2006; Merkel 2017). Ein anderer prominenter Ansatz sieht in der Flüchtlings- und Migrationspolitik westeuropäischer Nationalstaaten keine gänzlich neue Dimension des Parteienwettbewerbs, sondern ein weiteres Politikfeld, das - je nach gesellschaftlichen Voraussetzungen - in bestehende dominante Konfliktlinien integriert wird 
(Odmalm 2011). Flüchtlings- und Migrationspolitik kann demnach ideologisch sowohl einer wirtschaftlichen Wettbewerbsdynamik folgen, als auch aus einer gesellschaftspolitischen Dynamik, die sich wiederum mehr an postmaterialistischen versus materialistischen Werten angliedert, aber auch moralische Implikationen beinhalten kann. Jedes Land besitzt andere Voraussetzungen für die Parteien und lässt daher bei der Migrationsdebatte unterschiedliche Wettbewerbs- und Framingstrategien zu (Bale 2003; Boswell 2009b; Dahlström und Esaiasson 2013; Givens und Luedtke 2004; Green-Pedersen und Krogstrup 2008; Wayne et al. 1994).

Der vorliegende Beitrag nimmt an, dass sich die Parteien im Bundestag durch die Überlappung verschiedener Dimensionen im untersuchten Politikfeld entsprechend der allgemeinen Links-Rechts-Dimension positionieren, die je nach zeitlichem Themenschwerpunkt andere Wettbewerbsdynamiken begünstigt. Durch diese Strategie lässt sich eine ideologische Positionierung der Parteien von links nach rechts erwarten. Daten des Chapel-Hill-Expert-Survey bilden die allgemeine Links-RechtsDimension mit dem Item lrgen (left-right general) ab (Polk et al. 2017) ${ }^{2}$. Die Daten lassen ein Ranking der Parteien in Deutschland in aufsteigender Reihenfolge erwarten: Die Linke, Bündnis 90/Die Grünen, SPD und dann CDU sowie CSU (ebd.).

Hypothese 1 Die Parteipositionen in der deutschen Flüchtlingspolitik lassen sich auf einer allgemeinen politischen Dimension verorten. Daher ist die folgende Reihung von links nach rechts zu erwarten: Die Linke, Bündnis 90/Die Grünen, SPD und dann CDU sowie CSU.

\subsection{Parlamentarischer Parteienwettbewerb: Opposition und Regierung}

Einer der wohl bedeutendsten Faktoren für die Strukturierung des Parteienwettbewerbs in parlamentarischen Demokratien ist die Unterscheidung der Parteien nach Regierung und Opposition (Franzmann 2016). Ob eine Partei in Regierungsverantwortung ist oder nicht, bestimmt ihre Machtposition im Parlament und damit auch, welcher Mittel sie sich bedienen kann, um am legislativen Prozess mitzuwirken. Einer schwachen Opposition bleiben für die Umsetzung ihrer eigenen Interessen im Parlament nur begrenzte Strategieoptionen, da sie ausschließlich durch Kooperation mit den Regierungsparteien Impulse in Gesetzesvorlagen einbringen kann (Hohl 2017). Eine klare Konfrontationsstrategie ermöglicht zwar ein Gegengewicht im politischen Diskurs der Parlamentsparteien, verringert aber die Chancen eigene Gesetzesentwürfe mehrheitsfähig zu machen.

Die Opposition kann sich bei homogenen Positionen themenweise organisieren oder gespalten das Gegenstück zur Regierung bilden, wenn beispielsweise bei einer Regierung der Mitte am linken und rechten Ende der ideologischen Skala Oppositionsakteurinnen und -akteure positioniert sind oder gar eine Anti-System-Partei im Parlament vertreten ist. Im Falle des 18. Deutschen Bundestags gehören die Parteien Die Linke sowie Bündnis 90/Die Grünen zur Opposition und stehen der Regierung bei der Flüchtlings- und Migrationspolitik auf dem linken Ende der Skala

\footnotetext{
${ }^{2}$ Im Fragebogen des Chapel-Hill-Expert-Survey wird das Item mit der Beschreibung „Position der Partei im Jahr 2014 in Bezug auf ihre allgemeine ideologische Haltung“ beschrieben (Polk et al. 2017).
} 


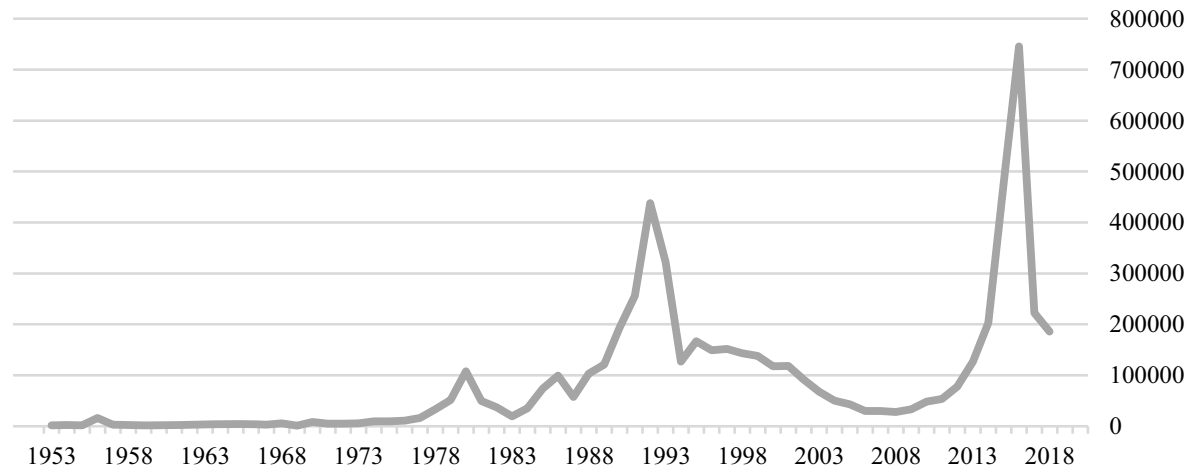

Abb. 1 Asylanträge (Erst- und Folgeanträge) nach Zahlen des BAMF zwischen 1953 und 2018. (Quelle: Bundesamt für Migration und Flüchtlinge 2019)

gemeinsam gegenüber. Die Aufgabe der Opposition ist vor allem die Kontrolle des Regierungshandelns. Diese Aufgabe stellt im 18. Bundestag eine besondere Herausforderung dar, da die Opposition mit 20,32\% der Sitze besonders klein ist und einige Kontrollfunktionen nur mithilfe von Stimmen aus den Regierungsparteien nutzen kann. Aus diesem Grund verabschiedete die Regierung eine Sonderregelung zum Minderheitenschutz, die die Hürden für beispielsweise die Einsetzung eines Untersuchungsausschusses heruntersetzt. Weitere Anstrengungen der Partei Die Linke, andere Hürden für die effektive Arbeit der Opposition an die Gegebenheiten anzupassen, wurden vom Bundesverfassungsgericht am 3. Mai 2016 abgewiesen (Bundesverfassungsgericht 2016).

Aus diesem Grund wird entsprechend der in H1 angenommenen Reihung, aber entgegen der Einschätzungen von Expertinnen und Experten (Polk et al. 2017), für die Arena des Parlaments eine deutliche Differenz zwischen den Positionen der Regierungs- und Oppositionsparteien angenommen. Die Regierungsparteien positionieren sich als Einheit und auch die Oppositionsparteien positionieren sich als Teil ihrer Abgrenzungsstrategie zur Regierung geschlossener, als es ihre ideologische Ausrichtung erwarten lässt. Besonders deutlich wird die Diskrepanz bei der SPD, die ideologisch laut Chapel-Hill-Expert-Survey fast dieselbe Position einnimmt wie Bündnis 90/Die Grünen, aber durch ihre Regierungszugehörigkeit an die Position der CDU/CSU-Fraktion rückt (ebd.).

Hypothese 2 Die Positionen der Oppositionsparteien und Regierungsparteien im Bundestag sind in den Positionen zur Flüchtlings- und Migrationspolitik deutlich different und lassen sich anhand geringerer Distanz der Positionen innerhalb der beiden Gruppen und einer großen Distanz zwischen ihnen messen.

\subsection{Die SPD zwischen Regierungsverantwortung und Überzeugung}

Nach der Konzeption des Politikfeldes sowie der Herausarbeitung der parteipolitischen und ideologischen Dynamiken im Parlament, ist es wichtig zu verstehen, warum die SPD in dieser Konstellation eine Sonderrolle als Regierungspartei und 

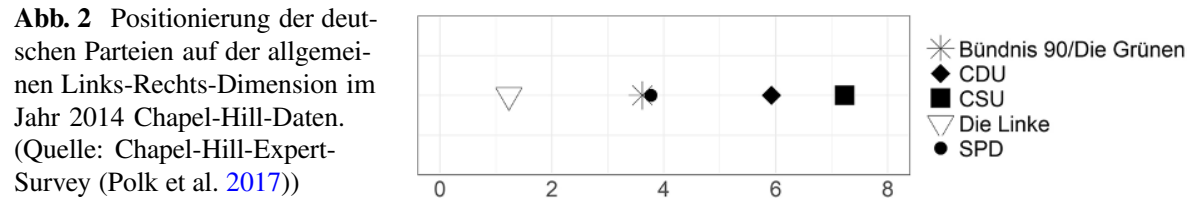

ideologisch mittig positionierte Partei einnimmt. Die SPD modernisierte sich ab den 1990ern zu einer Catch-All-Partei und geriet durch den Wettbewerb mit ideologischen Nachbarn aus beiden Lagern, sowohl von linker als auch rechter Seite, über die Jahre immer weiter unter Druck (Dostal 2017a). Der Parteienwettbewerb war in Deutschland von den beiden großen ehemaligen Volksparteien SPD und CDU dominiert, die in unterschiedlichen Koalitionskonstellationen oft gemeinsam die Regierung stellten (Niedermayer 2015, 2018). Aus der Zusammenarbeit in den Großen Koalitionen resultiert auf der sozio-ökonomischen Wettbewerbsdimension eine Linksverschiebung in Richtung mehr Staatsinterventionismus sowohl bei der SPD als auch bei der CDU. Zusätzlich vollzog die CDU auf der sozio-kulturellen Wettbewerbsdimension eine Verschiebung in die Mitte (Jakobs und Jun 2018; Jun 2019). Dies sowie die Etablierung neuer Parteien, wie der Alternative für Deutschland (AfD), und damit die flexibler werdenden Wettbewerbsdynamiken stellen die SPD vor Herausforderungen, besonders im hier untersuchten Politikfeld Migration während der 18. Legislaturperiode im Bundestag.

Die von der SPD 2011 ausgeschlossene Option auf eine Große Koalition und ihre daraus resultierenden Probleme bei der Koalitionsbildung mit der CDU/CSU 2013 sind ein weiteres Indiz für die angespannte Wettbewerbs- und Koalitionssituation der Partei (Dostal 2017a). Während der Koalitionsverhandlungen und dem anschließenden Mitgliedervotum stimmten die Jusos gegen die Fortsetzung der Großen Koalition (FAZ 2013; Spiegel Online 2013) und auch Wolfgang Thierse, langjähriger SPDPolitiker, beschreibt die Situation als Zerreißprobe für die SPD (Deutschlandradio 2013). Dennoch stimmen die Mitglieder der SPD mit $76 \%$ der Stimmen für die Große Koalition (Süddeutsche Zeitung 2013). Der Eintritt in die Regierungskoalition war mit dem Mitgliederbeschluss entschieden. Wie weit die SPD aber tatsächlich ideologisch von den anderen Regierungsparteien positioniert war, zeigen die Chapel-HillDaten (Abb. 2). Die SPD positionierte sich nach Einschätzungen von Expertinnen und Experten aus dem Jahr 2014 viel dichter zu den beiden Oppositionsparteien als zu den Parteien der Großen Koalition. Die ideologische Nähe der Parteien und die Bemühungen in den Koalitionsverhandlungen reichten für ein Regierungsbündnis und zeigen dennoch ein sehr gespaltenes Bild der SPD. Die ideologische Positionierung zur Opposition und die Verpflichtungen gegenüber den Regierungsparteien CDU und CSU stellen einen Zielkonflikt dar: die Verfolgung linkerer Positionen als die Regierungsparteien und die Teilhabe am Regierungsbündnis als handlungsfähige Partei, die ihre Entscheidungen gegenüber ihrer Wählerschaft rechtfertigen muss.

Darüber hinaus zeigt sich hier, dass besonders die SPD unter Druck steht, die Flüchtlings- und Migrationspolitiken der Regierung zu rechtfertigen. Das liberale Dilemma, demzufolge liberale Staaten vor allem in der Migrationspolitik mit Zielkonflikten konfrontiert sind (Hollifield 2015), stellt vor allem Regierungsparteien vor Herausforderungen. Die Handlungsmaximen des Staates und somit auch 
von Regierungsparteien lassen sich anhand von vier Zielen verdeutlichen: Fairness bei der Umverteilung, Akkumulation von Reichtum, Erhaltung eines hohen Maßes an Sicherheit für die Bevölkerung sowie Sicherung der institutionellen Legitimität (Boswell 2009b). Die Verfolgung eines dieser Ziele bedeutet auch immer, Abstriche in einem der anderen hinnehmen zu müssen (ebd.). Dieses Dilemma wird für die liberalere SPD dadurch verstärkt, dass sie grundsätzlich eine deutlich linkere Position als die anderen Regierungsparteien verfolgt. Daraus lässt sich die Erwartung ableiten, dass sich die SPD relativ zur Union sehr unterschiedlich positioniert - abhängig davon, ob sie eher ihrer ideologischen Ausrichtung oder ihren Verpflichtungen innerhalb der Regierungskoalition folgt. Sie ist als einzige Partei mit einer Vielzahl an Dilemmata konfrontiert, die ihre Handlungsfähigkeit in der Flüchtlings- und Migrationspolitik beeinflussen. Neben der Regierungsverantwortung sollte daher auch der thematische Schwerpunkt bzw. die Betonung unterschiedlicher inhaltlicher Facetten des Politikfeldes einen Einfluss auf die Position der SPD haben. Die SPD befindet sich in einem starken Spannungsverhältnis zwischen ihren Koalitionsverpflichtungen und ihrer eigenen ideologischen Positionierung:

Hypothese 3 Die Position der SPD zur CDU gestaltet sich im Laufe der Legislaturperiode ungleichmäßig, d.h. sie positioniert sich mal entsprechend ihrer Regierungszugehörigkeit dichter an der CDU oder, entsprechend ihrer eigentlichen ideologischen Positionierung, dichter an den Oppositionsparteien.

\section{Daten und Methoden}

Der verwendete Datensatz besteht aus Reden der Parlamentarierinnen und Parlamentarier des Deutschen Bundestages, die in der 18. Legislaturperiode zur Flüchtlingsund Migrationspolitik mit den Stichworten „Flüchtling“ und „Migration“ gesprochen haben. Ausgewählt wurden die Reden durch den Suchalgorithmus des Archivs des Deutschen Bundestags sowie einer manuellen thematischen Korrektur. Mithilfe des Suchalgorithmus werden zunächst 163 Debatten ausgewählt und nach einer weiteren Auswahl 62 analyserelevante Debatten, bestehend aus 312 Reden, zu einem Datensatz von 186.904 Wörtern zusammengefügt (siehe Tab. 2).

Zunächst wird der Datensatz bereinigt (Miles 2009; Porter und Bolton 2001). ${ }^{3}$ Ausgeschlossen werden Worte, die nicht mindestens von zwei Akteurinnen oder Akteuren verwendet werden, da sie nicht zur Bestimmung der ideologischen Distanz zwischen ihnen verwendet werden können. Idealerweise sollte jedes Analysewort von jeder Akteurin und jedem Akteur verwendet werden (Proksch und Slapin 2010). In dieser Analyse wird die Verwendung der Wörter von mindestens zwei Parteien als Kriterium gesetzt. Ein ganz praktischer Grund hierfür ist außerdem, dass Wordfish erst ab 800-1000 Wörtern pro Analysetext stabil valide Ergebnisse berechnen kann

\footnotetext{
3 Zur Erhöhung der Vergleichbarkeit werden die Texte vor der Analyse einer Lemmatisierung unterzogen. Des Weiteren werden Stoppwörter entfernt. Angelehnt an die Stoppwörterliste des Snowball-StemmingAlgorithmus wurde die Auswahl der Wörter analysespezifisch erweitert. Schließlich werden noch Zahlen und Währungssymbole entfernt und der gesamte Text klein geschrieben.
} 


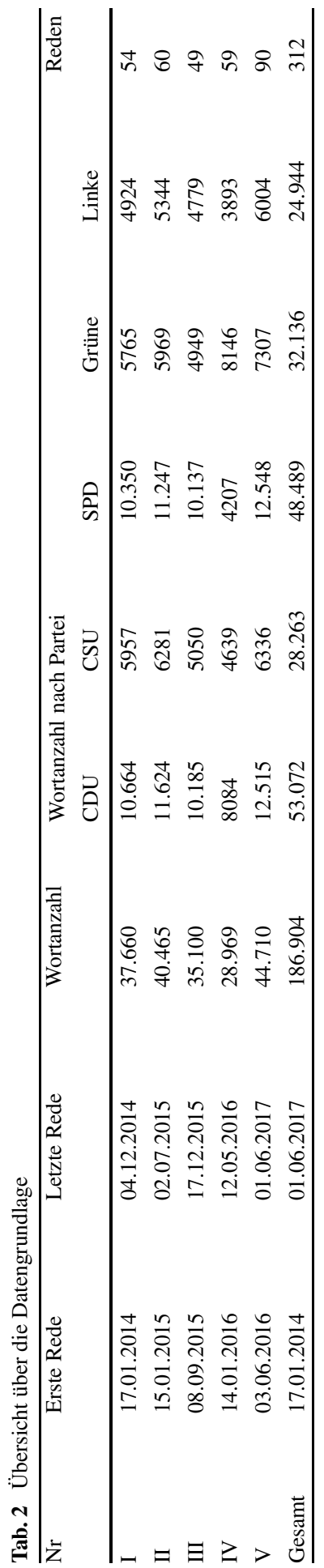


(ebd). Ein zu striktes Kriterium würde die Menge der zu analysierenden Wörter zu stark minimieren (Frid-Nielsen 2018; Slapin und Proksch 2008). Dies kann durch einen angemessenen Zuschnitt der Analysezeiträume aufgefangen werden (siehe Tab. 3). Grundsätzlich gilt: Je mehr Wörter in den untersuchten Analysedokumenten vorhanden sind, desto besser werden die Positionsschätzungen. Dies ist nicht nur methodisch relevant, sondern auch theoretisch gut herzuleiten (Lo et al. 2016). Lange Statements oder Reden ermöglichen eine differenzierte Darlegung von Positionen und die Verwendung von ideologisch relevanten Wörtern, die der Argumentation Nachdruck verleihen und von Wordfish für die Berechnung der Position verwendet werden.

Die Betrachtung der Rolle der Politikerinnen und Politiker, die die Plenardebatten halten, führen zu einer weiteren analyserelevanten Überlegung: Die Aggregation der Rednerinnen- und Rednerdaten auf Parteiebene. Für das Forschungsinteresse an Parteipositionen ist dies notwendig und gewährleistet die von Wordfish benötigten Textlängen. Des Weiteren erhalten u. a. Proksch und Slapin (2010) mit diesem Vorgehen bei ihrer Analyse des europäischen Parlaments angemessen valide Ergebnisse und zeigen außerdem, dass die Analyse bei sorgfältiger Datenaufbereitung robust gegen das Wegfallen einzelner Debatten ist.

Um die Problematik der geringen Worthäufigkeiten aller Parteien zu einem Debattenzeitpunkt zu umgehen, wird der Beobachtungszeitraum in fünf vergleichbare Abschnitte unterteilt. Neben valideren Messergebnissen kann dadurch die Analyse verschiedener Dynamiken in den Untersuchungszeiträumen vorgenommen werden. So zeichnen sich die Zeiträume durch ganz eigene thematische Schwerpunkte und daraus resultierende Wettbewerbsdynamiken aus.

Ausgewählt werden der Start der Legislaturperiode sowie ab Januar 2015 die deutlich ansteigende Asylantragszahlen. Im Analysezeitraum I debattiert das Parlament vor allem über Themen der Außenpolitik: die Einstufung weiterer Länder als sichere Herkunftsländer, um die Bearbeitung der Asylanträge aus diesen Regionen $\mathrm{zu}$ beschleunigen sowie das Vorgehen bei der Seenotrettung im Mittelmeer. Der Analysezeitraum I beinhaltet 54 Reden und besteht aus 37.660 Wörtern. Der Analysezeitraum II besteht aus 60 Debatten und 40.465 Wörtern. Hier liegt der inhaltliche Fokus der Debatten auf dem Einwanderungsgesetz und der damit verbundenen lokalen Integrationspolitik. Grundsätzlich ist die Zeit von einer positiven Stimmung der Willkommenskultur geprägt.

Nach der ,Wir-schaffen-das“-Rede von Angela Merkel beginnt der Analysezeitraum III, der 49 Reden beinhaltet und aus 35.100 Wörtern besteht. Die parlamentarische Debatte dreht sich vor allem um die Einführung eines Asylverfahrensbeschleunigungsgesetzes, da die Überlastung der Behörden immer mehr Konsequenzen für die eingereisten Flüchtlinge hat (FAZ 2015) und medial negativ diskutiert wird (Spiegel Online 2015a; welt.de 2015). Die Regierungsparteien stehen unter Zugzwang, die anspannte Situation zu entlasten. Mit der Kölner Silvesternacht 2015/2016 beginnt ein neuer Abschnitt in der Debatte. Der Analysezeitraum IV umfasst 59 Debatten und besteht aus 28.969 Wörtern. Die Debatte wird polarisierter, da die Übergriffe in der Silvesternacht als Beispiel für die Überforderung der Behörden instrumentalisiert werden (NDR 2016). Der in den Medien stark thematisierte Bruch zwischen CDU/CSU ist der Startpunkt für den letzten Analysezeitraum (Süddeutsche Zeitung 


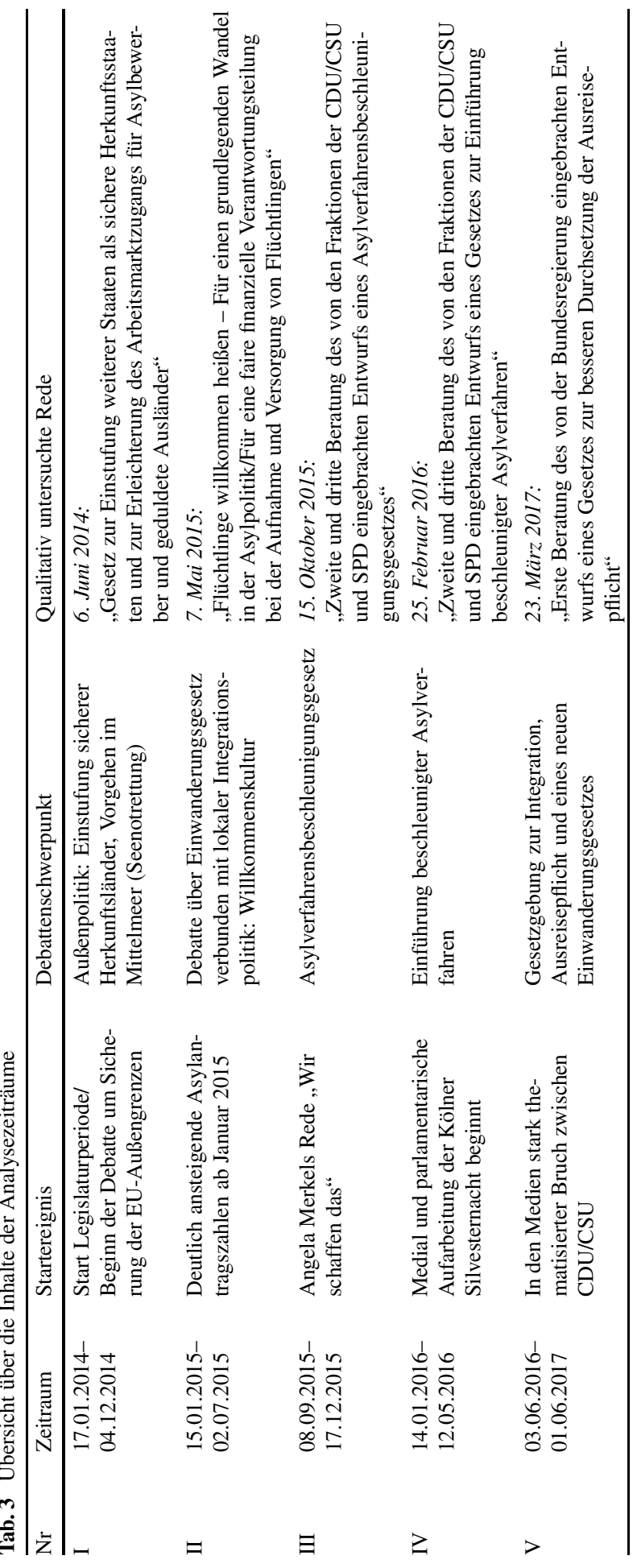


2016a), der aus 90 Debatten und 12.515 Wörtern besteht. Die abfallenden Asylantragszahlen sowie die erfolgreich verabschiedeten Asylpakte I und II entspannten die politische Debatte nur im geringen Maße, da die CDU/CSU-Fraktion interne Streitigkeiten öffentlich austrägt (Handelsblatt 2018). Im Parlament verschiebt sich die Debatte auf andere Themenkomplexe der Migrationspolitik. So werden die Integrationsgesetzgebung sowie ein Gesetz zur Durchsetzung der Ausreisepflicht und die Konzeption eines neuen Einwanderungsgesetzes besprochen.

Zur Bestimmung der Parteipositionen wird das quantitative Textanalyseprogramm Wordfish verwendet. Die Software besitzt gegenüber anderen quantitativen Textanalyseverfahren wie Wordscores den klaren Anwendungs- und Skalierungsvorteil, ohne Ankertexte vorzugehen, d.h. es können auch Texte analysiert werden, bei denen ein geringes Vorwissen über die Positionierung vorhanden ist (Slapin und Proksch 2008). Neben der Analyse ohne notwendige Vorannahmen eignet sich Wordfish für einen explorativen Umgang mit Daten, der durch die Aufdeckung von Mustern die Grundlage für weitergehende Analysen bilden kann. Die Arbeit mit wenigen Vorannahmen bedeutet auch, dass eine Validierung der Ergebnisse im Nachhinein erfolgen muss, da Wordfish sonst zu erheblichen Validitätsproblemen führen kann (Dolezal et al. 2016). Dies wird durch die sorgfältige Zusammensetzung der Analysezeiträume, die interne Validitätsprüfung anhand der vergebenen Wortgewichte (Abbildung A-1 und Tabelle A-2 im Online-Zusatzmaterial) sowie eine externe Validitätsprüfung mithilfe der qualitativen Analyse einzelner Debatten kontrolliert.

Slapin und Prokschs (2008) Wordfish ist auf Grundlage der Item Response Theory modelliert, um automatisiert Positionen innerhalb eines eindimensionalen Raums zu lokalisieren (Grimmer und Stewart 2013). Es wird angenommen, dass sich jede Politikerin und jeder Politiker $i$ als Vertreterin ihrer und Vertreter seiner Partei auf einer skalierbaren Dimension positioniert, die in dem Modell durch den Parameter $\theta$ beschrieben wird. Diese latente Position beeinflusst die Verwendung der Wörter, also die relativen Worthäufigkeiten, anhand derer Wordfish die ideologische Position in Relation zu anderen in der Analyse berücksichtigten Positionen schätzt.

Überdies beeinflusst nicht nur die ideologische Überzeugung der Akteurinnen und Akteure ihre Worthäufigkeiten, sondern auch diskursive Regeln, wie das Issue Ownership oder im parlamentarischen Kontext beispielsweise die Redezeiten, die den Akteurinnen und Akteuren für ihre Äußerung zur Verfügung stehen. Issue Ownership bedeutet hier, dass Parteien bestimmte Themenbereiche im politischen Diskurs und Wettbewerb für sich beanspruchen und versuchen, sich in ihnen zu spezialisieren, um sich bei der Wählerschaft mit Kompetenzbereichen als attraktiv und wählbar zu präsentieren (Petrocik 1996). Dies ist ebenso in den parlamentarischen Kontext übertragbar, da sich diese thematische Spezialisierung als grundsätzliche Wettbewerbsstrategie bewährt hat. Es ist für die Akteurinnen und Akteure einfacher, sich im Parteienwettbewerb zu behaupten, anstatt in ein- und demselben Themenbereich mit einer anderen Partei in direkte Konkurrenz zu treten. Durch Issue Ownership verwenden verschiedene Politikerinnen und Politiker oder Parteien auch im Diskurs in einem Themenbereich ein hoch spezialisiertes und ideologisch aufgeladenes Vokabular, das stark von ihren Wettbewerberinnen und Wettbewerbern abweicht und so zu ihrer Unterscheidung verwendet werden kann. Zusätzlich trägt die stark 
formalisierte Verteilung der Redezeiten zu einer systematischen Beeinflussung des verwendeten Vokabulars bei.

Die von Wordfish bestimmte Dimension wird in den fünf bestimmten Analysezeiträumen durch verschiedene Validierungsprozesse nachbereitet. Zunächst wird die interne Validität durch die Abbildung einer Wortwolke getestet. Wordfish vergibt für jedes Analysewort einen Beta- und einen Psi-Wert ${ }^{4}$. Diese lassen sich anhand eines Diagramms abbilden und zur Prüfung der internen Validität der Messung nutzen. Proksch und Slapin (2008) verweisen bei der Feststellung der Güte der internen Validität auf die „Eiffelturm-Form“ der Wortwolke. Je deutlicher die Form des Eiffelturms zu sehen ist, desto mehr gemeinsames Vokabular haben die Akteurinnen und Akteure verwendet, was für eine verbindende Dimension der bearbeiteten Texte spricht. Je ausgeprägter die „Füße des Eiffelturms“, desto spezifischer ist das Vokabular, das die Akteurinnen und Akteure einer ideologischen Ausrichtung verwenden und anhand derer sie dieser durch das Programm zugeordnet werden können (ebd.).

Neben dieser optischen Diagnosemethode können die Wörter, die niedrige BetaWerte und extreme Psi-Werte besitzen, helfen, die inhaltlichen Schwerpunkte der analysierten Debatten zu bestimmen. Diese werden außerdem für die Auswahl der Debatten verwendet, die der qualitativen Analyse unterzogen werden.

Die Auswahl der richtigen Debatten ist nicht nur thematischer Natur, sondern beeinflusst maßgeblich die von Wordfish bestimmte Diskursdimension, auf der die Parteien positioniert werden. Grundsätzlich muss die von Wordfish bestimmte Diskursdimension ex post bestimmt werden. Diese Eigenschaft von Wordfish bedeutet für die Datenaufbereitung, dass die Texte und Positionen nur valide miteinander verglichen und als zeitliche Entwicklung betrachtet werden können, wenn sie zum selben Diskurs gehören. Die ausgewählten Parlamentsreden haben daher neben denselben thematischen Schwerpunkten gemeinsam, dass sie in einem recht eng gefassten Zeitraum zu denselben Ereignissen im selben Kontext Stellung nehmen, sodass beispielsweise der Bedeutungswandel von Wörtern über Dekaden vernachlässigbar ist. Nur bei einer sorgsamen Datenaufbereitung kann bei diesem Mixed-MethodsAnsatz am Ende von Positionen zu Flüchtlings- und Migrationspolitik gesprochen werden.

Als Ergänzung zu den Wordfish-Ergebnissen wird pro Analysezeitraum eine Rede mithilfe einer qualitativen Inhaltsanalyse mit induktiver Kategorienbildung sowie analyseleitendem Selektionskriterium analysiert (Mayring 2015). Dies hat zum Ziel, die Argumente der unterschiedlichen Parteien, die als Selektionskriterium der Inhaltsanalyse dienen, besser nachvollziehen und die Dynamiken bzw. Veränderungen in den Argumenten einzelner Parteien nachverfolgen zu können. Ausgewählt werden die Reden anhand der von Wordfish im Analysezeitraum gefundenen Diskursdimension. Diese lässt sich anhand der von Wordfish vergebenen Beta- und PsiWerte ableiten. Eine Übersicht der Beta- und Psi-Werte sowie der Ergebnisse der

\footnotetext{
${ }^{4}$ Jedes Wort erhält einen Beta- und einen Psi-Wert. Beta sind die Worttypgewichte (Word Weights) und Psi die Worttypeneffekte (Word Fixed Effects). Niedrige Beta-Werte und niedrige Psi-Werte zeigen demnach die Wörter an, die für das linke Skalenende charakteristisch sind, hohe Beta-Werte und niedrige Psi-Werte für das rechte Skalenende. Beta-Werte um Null sowie hohe Psi-Werte beschreiben Wörter, die von allen Akteurinnen und Akteuren gleichermaßen verwendet werden und nicht zur ideologischen Unterscheidung der Positionen verwendet werden können.
} 
qualitativen Inhaltsanalyse finden sich im Online-Anhang (Tabelle A-2 im OnlineZusatzmaterial). Neben dem tieferen Einblick in die Diskursdimension ist durch die qualitative Analyse eine externe Validierung der Wordfish-Ergebnisse möglich.,

\section{Empirische Analyse}

Zunächst werden für den gesamten Zeitraum vom 17. Januar 2014 bis zum Ende der Legislaturperiode und der letzten Rede am 1. Juni 2017 mit Wordfish die Positionen der Parteien bestimmt (Benoit et al. 2018) ${ }^{7}$. Wie der Abb. 3 zu entnehmen ist, positionieren sich vom linken ideologischen Ende Die Linke, dann Bündnis 90/Die Grünen, die SPD und dann die CDU und weiter rechts die CSU. Dies entspricht den Annahmen aus Hypothese 1. Schon hier lässt sich die in Hypothese 2 formulierte Distanz zwischen den Oppositions- und Regierungsparteien feststellen. Außerdem ist innerhalb der Regierungsparteien die ideologische Distanz zwischen den beiden Schwesterparteien CDU und CSU am geringsten und auch die beiden Oppositionsparteien positionieren sich dicht zu einander. Die SPD positioniert sich in dieser Gesamtanalyse entfernt von den anderen Regierungsparteien und trotzdem in deutlicher Distanz zu den Oppositionsparteien. Inwiefern diese Gesamtpositionierung der SPD das Ergebnis variierender Positionierungen zu unterschiedlichen Zeitpunkten ist, wie von Hypothese 3 erwartet, wird im nächsten Schritt der empirischen Analyse untersucht.

\subsection{Positionsbestimmung in den Analysezeiträumen}

In allen fünf Analysezeiträumen positionieren sich die Parteien in derselben Reihung, wie in Hypothese 1 angenommen wird (Abb. 3, Tabelle A-1 im OnlineZusatzmaterial). Es gibt jedoch interparteiliche Differenzen in den Distanzen der Positionen über den Zeitverlauf. ${ }^{8}$ In allen fünf Analysezeiträumen positioniert sich, wie in Hypothese 2 angenommen, die Opposition sehr geschlossen und auch die

\footnotetext{
5 Die Akquirierung der Plenarprotokolle stellt eine Herausforderung dar. Werden Debatten zu bestimmten Themenschwerpunkten wie zur Flüchtlings- und Migrationspolitik gesucht, werden diese mithilfe einer Suchmaschine selektiert. Fehler in deren Algorithmus sind jedoch meist nur schwer zu finden und auch die Genauigkeit der Zuordnung zu dem gewählten Bereich ist nicht immer wie gewünscht, sodass die Debatten nach der Auswahl durch den Suchalgorithmus des Deutschen Bundestags manuell auf ihre Passgenauigkeit zum gesuchten Politikfeld überprüft werden. Hierdurch werden die Daten deutlich verkleinert und in ihrer Qualität verbessert.

6 Die qualitativ herausgearbeiteten Argumente (Tabellen A-3 bis A-7 im Online-Zusatzmaterial) ergänzen die Wordfish-Ergebnisse. Diese ermöglichen die räumliche Verortung der Parteien auf einer Dimension, aber können keine argumentative Verbindung zwischen den analysierten Wörtern herstellen. Dies leistet die qualitative Analyse und zeigt darüber hinaus argumentative Allianzen und Dynamiken im Parteienwettbewerb.

7 Dieselben Muster finden sich auch bei der Durchführung einer eindimensionalen Korrespondenzanalyse (Abbildung A-2 und Tabelle A-12 im Online-Zusatzmaterial).

8 Die Parteipositionen können nicht zwischen den einzelnen Analysezeiträumen miteinander verglichen werden, da die Skalen nicht identisch sind. Es lässt sich aber die relative Distanz zwischen den Parteien vergleichen und Aussagen über die Dynamik des Parteienwettbewerbs treffen.
} 

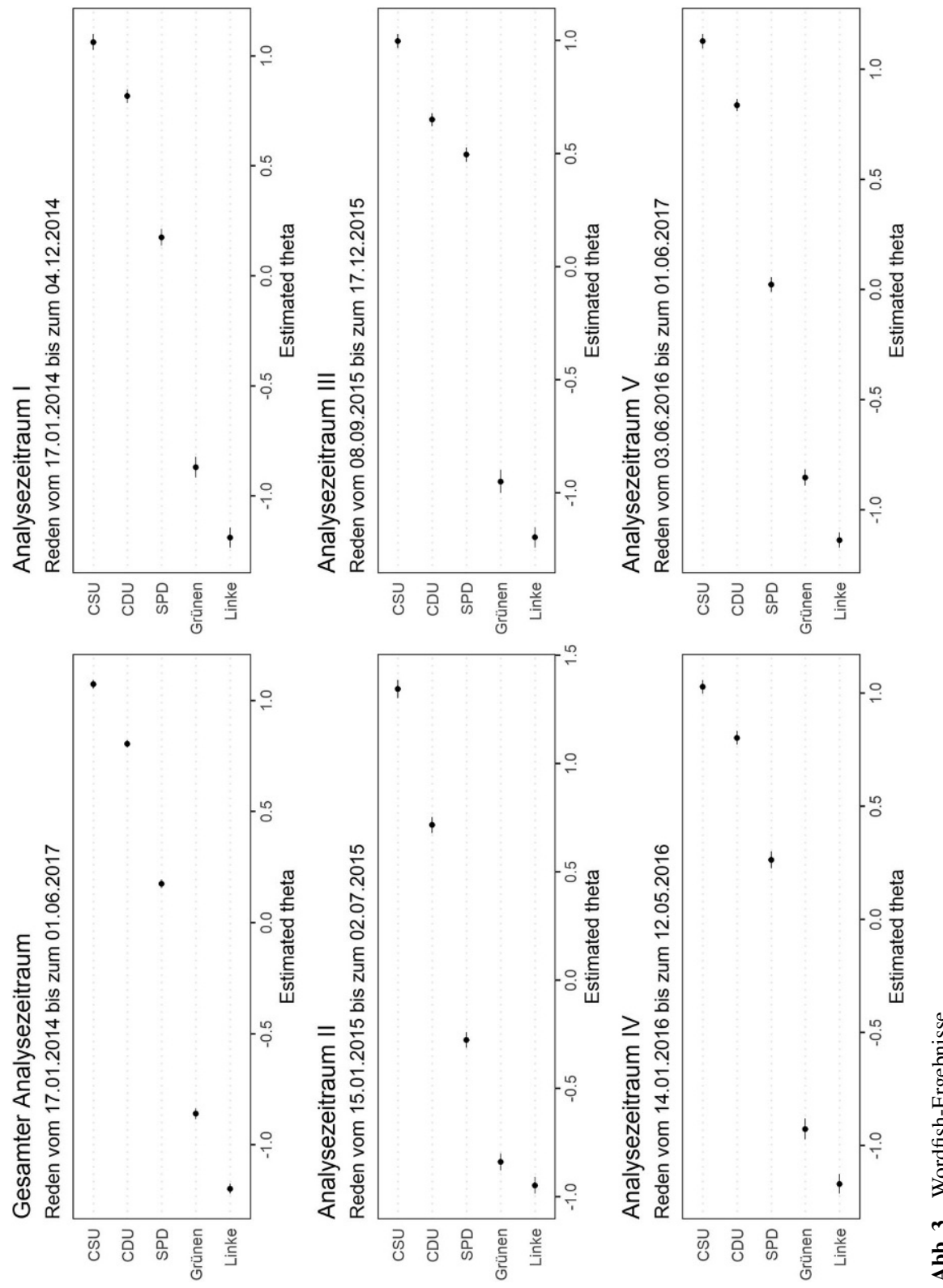

Schwesterparteien CDU und CSU befinden sich, wie erwartet, gemeinsam am entgegengesetzten ideologischen Ende der Skala. Wie in Hypothese 3 angenommen positioniert sich die SPD dynamisch. Im zweiten und im letzten Zeitraum befindet sie sich im Gegensatz zu den anderen Analyseeinheiten distanziert von den Regierungsparteien. Im zweiten gruppiert sie sich zur Opposition und im fünften 
Analysezeitraum eher in der Mitte mit gleichmäßigem Abstand zur Opposition und den Regierungsparteien.

Die genauen Wortverteilungen können dem Anhang entnommen werden und dienen an dieser Stelle der deskriptiven Analyse der Diskursschwerpunkte, die neben den relativen Positionsbestimmungen einen tieferen Einblick in die Diskursinhalte und relativ stabilen Wettbewerbsdynamiken geben (Tabelle A-2 im Online-Zusatzmaterial). Im gesamten Zeitraum und auch bei den fünf Analysezeiträumen sind die neutralen Wörter der Debatte größtenteils identisch. Die Verwendung der Begriffe „mensch“, „flüchtling“, „,deutschland“, „land“, „länder“, ,euro(pa)“, oder ,europäisch“ zeigen, dass der zusammengestellte Datensatz migrations- und vor allem flüchtlingspolitische Debatten beinhaltet und dass diese Dimension stabil sowohl im gesamten Zeitraum als auch in den einzelnen Analysezeiträumen zu finden ist.

\subsection{Thematische Schwerpunkte in den Analysezeiträumen}

In Analysezeitraum I sieht sich die Politik mit ansteigenden Zahlen von Flüchtlingen in Deutschland konfrontiert. Die Debatte im Bundestag wird vor allem von außenpolitischen Themen, wie der Einstufung sicherer Herkunftsländer (Tabelle A-3 im Online-Zusatzmaterial) und dem Vorgehen im Mittelmeer, genauer der Seenotrettung, bestimmt. Sowohl die Wordfish-Ergebnisse als auch die qualitative Analyse zeigen, dass die Oppositionsparteien an die Wahrung der Menschenrechte und -würde appellieren (,schande“, ,,völkerrechtswidrig“) und die Einstufung weiterer Länder als sichere Herkunftsstaaten ablehnen. Die Regierungsparteien stehen relativ geschlossen und deutlich näher zueinander positioniert. Die SPD teilt ihre Argumentation darin weitesgehend, dass das Asylrecht in Deutschland ,wirklich Schutzbedürftigen“ vorbehalten sein soll (,tatsächliche“, ,,aussichtslose“ [Asylanträge]). Und vor allem die CDU/CSU-Fraktion spricht sich aufgrund dieser Logik für eine Ausweitung der Anerkennung weiterer Staaten als sichere Herkunftsländer aus (Tabelle A-3 im Online-Zusatzmaterial). Die Nähe der Regierungsparteien im ersten Analysezeitraum lässt sich mit dem Start der Legislaturperiode und den zeitlich naheliegenden Koalitionsverhandlungen erläutern. Auch der thematische Fokus des ersten Analysezeitraums auf außenpolitische Fragen und dortige Übereinstimmungen in den politischen Forderungen zwischen den Regierungsparteien könnte die Dynamik erklären.

Im Analysezeitraum II bewegt sich die SPD deutlich auf die Oppositionsparteien zu. Der Diskurs ist von den steigenden Asylantragszahlen Anfang 2015 geprägt sowie dem Diskurs über die Willkommenskultur und die Belastung der Kommunen durch die Aufnahme der Flüchtlinge (,,kommunen“, „,bund“). Die CDU/CSU-Fraktion betont in dem Zeitraum vor allem die bereits bereitgestellten Leistungen bei der Aufnahme der Flüchtlinge und argumentiert für eine Regulierung der Zuwanderung (,schutzgrund“, „fachkräfte“). Die Oppositionsparteien sprechen von katastrophalen Zuständen in den Aufnahmelagern und rekurrieren immer wieder auf die Menschenwürde, die trotz der Überforderung der Behörden für die Flüchtlinge gewahrt werden müsse (,,menschenwürde“, ,,inakzeptabel“). Besonders Bündnis 90/Die Grünen und die SPD teilen hier Argumentationslinien: Die Regierung müsse an einer europäischen Lösung der Migrationskrise und an sicheren Fluchtrouten arbeiten; daher sei 
die Zusammenarbeit des Bundes mit den Kommunen trotz der Zuständigkeit der Länder unabdingbar (Tabelle A-4 im Online-Zusatzmaterial).

Im Analysezeitraum III und IV positioniert sich die SPD dichter an ihren Koalitionsparteien am rechten ideologischen Ende des Spektrums. In den Zeiträumen wird über das Asylverfahrensbeschleunigungsgesetz sowie das Gesetz zur Einführung beschleunigter Asylverfahren debattiert und abgestimmt (Tabelle A-11 im Online-Zusatzmaterial). In der qualitativen Analyse zeigen sich in den beiden Debatten besonders der Koalitionszwang und die parteiinternen Zweifel der SPD an den im Gesetz formulierten Maßnahmen. So teilt die SPD wenige Argumente mit der CDU/CSU-Fraktion, kommentiert aber deren Argumente und relativiert zu einem gewissen Maße die eigene Position (Tabellen A-5 und A-6 im Online-Zusatzmaterial). Daher verwendet sie zwar ähnliches Vokabular - was die Wordfish-Ergebnisse erklärt -, die qualitative Analyse aber zeichnet ein differenziertes Bild: Die Sprecherinnen und Sprecher der SPD rechtfertigen die Zustimmung zum vorgebrachten Gesetzentwurf als notwendiges Mittel der Regierungsarbeit ohne die Diskrepanz zu ihren ideologischen Überzeugungen in den Fokus ihrer Argumentation zu stellen. Dies illustriert besonders den parlamentarischen Regierungs- und Oppositionskonflikt. In den beiden Analysezeiträumen sind die Flüchtlingszahlen auf ihrem höchsten Stand (Abb. 1) und die Überforderung der Behörden ist nicht nur medial präsent (FAZ 2015). Das rechte Ende der gefunden Diskursdimension rekurriert auf die „belastunsgsgrenze“ sowie eher technische Aspekte, wie die „beweislastumkehr“ (Tabelle A-2 im Online-Zusatzmaterial), während die Oppositionsparteien am linken Rand des Spektrums die ,abschottungspolitik“ kritisieren. Die Linke fordert darüber hinaus eine Altfallregelung, wie auch schon im Zeitraum II (Tabelle A-4 im Online-Zusatzmaterial).

Das Asylverfahrensbeschleunigungsgesetz sowie das Gesetz zur Einführung beschleunigter Asylverfahren sind in den beiden Analysezeiträumen III und IV hoch umstritten und werden insbesondere von der Opposition stark angegriffen. Dies zeigt sich beim Abstimmungsverhalten am 15. Oktober 2015 (Deutscher Bundestag 2015b). Wie der Tabelle im Anhang zu entnehmen ist, stimmt die Oppositionspartei Die Linke mehrheitlich gegen das Gesetz, Bündnis 90/Die Grünen enthält sich größtenteils und die Regierungsparteien stimmen dem Entwurf überwiegend zu, wobei CDU/CSU geschlossen dafür stimmen (Tabelle A-11 im Online-Zusatzmaterial). Einzig bei den Artikeln 8 des Finanzausgleichsgesetzes und 12 der Änderung des Entflechtungsgesetzes stimmen Bündnis 90/Die Grünen für die Vorlage der Regierungsparteien, da sie eigene Anliegen in diesen Gesetzesvorlagen verwirklicht sehen und durch dieses kooperative Verhalten unterstreichen wollen (Deutscher Bundestag 2015a, S. 3). Zusätzlich zu dieser Abstimmung und zum Inkrafttreten des Asylpakets I (Asylverfahrensbeschleunigungsgesetz) am 23. Oktober 2015, gab es am 25. Februar 2016 Abstimmungen zum Asylpaket II (Gesetz zur Einführung beschleunigter Asylverfahren), das am 17. März 2016 in Kraft getreten ist (Deutscher Bundestag 2016). In diesem Abstimmungsverhalten kann dann, zusätzlich zur Geschlossenheit der Oppositionsparteien, eine Uneinigkeit in den Reihen der SPD beobachtet werden.

Vom Analysezeitraum III, über IV und schließlich im Analysezeitraum V zeigt die Wordfish-Analyse ein graduelles Abrücken der SPD von den Regierungspar- 
teien hin zu den Oppositionsparteien (Abb. 3). Im fünften Analysezeitraum steht sie etwa in der ideologischen Mitte. Dies passt zu den Erwartungen aus Hypothese 3. Die Bewegung der SPD im Analysezeitraum III und IV lässt sich durch den Höhepunkt der Flüchtlingskrise und den in der Zeit starken politischen und medial eingeforderten Handlungsbedarf erklären (Haller 2017). Zwischen Juni 2016 und 2017 (Analysezeitraum IV) ist die mediale Berichterstattung von einem Bruch zwischen der CDU und CSU geprägt (Süddeutsche Zeitung 2016a). Im Bundestag wird über eine Anpassung der Integrationsgesetze, die Durchsetzung der Ausreisepflicht sowie die Konzeption eines neuen Einwanderungsgesetzes debattiert. Der Bundestag verabschiedete darüber hinaus am Anfang des Analysezeitraums V ein neues Integrationsgesetz. Die Union und SPD stimmten für das Gesetz, die Oppositionsfraktionen Die Linke und Bündnis 90/Die Grünen dagegen. Bestimmt wird der Zeitraum aber durch die anderen beiden Themen, was auch den Abstand der SPD zur CDU/CSU-Fraktion erklärt. So teilt die SPD in der analysierten Rede vom 25. Februar 2016 Argumente mit Die Linke und bedient nur bedingt die Argumente der anderen Regierungsparteien (Tabelle A-6 im Online-Zusatzmaterial).

\section{Diskussion der Ergebnisse und Ausblick}

Dieser Beitrag analysierte die Parteienpositionen im Bundestag zur Zeit der Flüchtlingskrise und konnte vor allem an der sich verändernden Position der SPD das Spannungsverhältnis zwischen der ideologischen Positionierung der Partei und dem auferlegten Koalitionszwang der Regierungsparteien aufzeigen. Darüber hinaus ermöglichte die Analyse tiefere Einblicke in die Dynamiken des facettenreichen Politikfeldes Flucht und Migration im Deutschen Bundestag sowie den Einfluss der parlamentarischen Arena auf die Diskursdynamiken.

An dieser Stelle sollen die Ergebnisse methodisch diskutiert und inhaltlich eingeordnet werden. Zunächst lässt die deskriptive Natur der verwendeten Analysemethoden nur limitiert Aussagen über kausale Zusammenhänge zu. Jedoch geben die gefundenen Dynamiken Anlass, die Wettbewerbskonstellationen während der Flüchtlingskrise zu reflektieren und die besondere Position der SPD im herausfordernden Politikfeld Flucht und Migration einzuordnen. Darüber hinaus zeigt die Analyse, dass sich mit Wordfish anhand fester Regeln mit parlamentarischen Debatten aus dem Deutschen Bundestag valide und reliable Parteipositionen bestimmen lassen. Dieser Mixed-Methods-Ansatz bietet durch die Vergabe von Wortgewichten, die bei einem qualitativen Blick in die Debattenschwerpunkte Orientierung geben, einen tieferen Einblick in die diskursbestimmenden Themenschwerpunkte, die sich in der Flüchtlings- und Migrationspolitik je nach Analysezeitraum verschieben. Die qualitative Analyse ermöglichte es darüber hinaus, die Argumentationen der Parteien und dadurch die Wettbewerbsdynamiken nachzuvollziehen. Mit der Betrachtung der unterschiedlichen Zeiträume konnte untersucht werden, ob das Spannungsverhältnis zwischen ideologischer Ausrichtung und Koalitionsräson, in dem sich die SPD befand, tatsächlich die erwarteten Auswirkungen auf ihre parlamentarische Positionierung hatte. 
So lässt sich die Hypothese 1 mit den analysierten Daten bestätigen. Die Parteien in Deutschland deuten die Flüchtlings- und Migrationspolitik anhand einer allgemeinen Links-Rechts-Dimension und positionieren sich in der vermuteten Reihenfolge in allen untersuchten Analysezeiträumen. Die beobachteten Ergebnisse zeigen die Stabilität des Parteienwettbewerbs im Deutschen Bundestag. Es lassen sich keine starken Effekte oder deutlichen Positionsveränderungen beobachten, jedenfalls nicht während der 18. Legislaturperiode. Weder der Erfolg der AfD nach ihrer Gründung 2013 noch der Wahlkampf auf Landesebene oder andere in der Öffentlichkeit als weitreichende Ereignisse in der Flüchtlingskrise wahrgenommenen Ereignisse scheinen einen Einfluss auf die Parteipositionen zu haben.

Der in Hypothese 2 formulierte Zusammenhang über das Positionsverhältnis von Regierung und Opposition lässt sich in allen Analysezeiträumen bestätigen. Die Positionen der Oppositions- und Regierungsparteien sind in den Ergebnissen der Analyse deutlich different und lassen sich anhand geringerer Distanz der Positionen innerhalb der beiden Gruppen und einer großen Distanz zwischen ihnen messen. Somit hat die Struktur der parlamentarischen Arena deutlichen Einfluss auf die Positionierung der Parteien innerhalb des Politikfelds Flucht und Migration, in dem die Trennung der Oppositions- und Regierungsparteien im Bundestag prominenter ausgeprägt ist, als bei der ideologischen Reihung auf Grundlage des Chapel-HillExpert-Survey, der eine allgemeine Einschätzung der Parteienpositionen vornimmt (Polk et al. 2017).

Der in Hypothese 3 formulierte Zielkonflikt der SPD zwischen ideologischer Ausrichtung und Koalitionsräson lässt sich ebenfalls beobachten. Bemerkenswert ist dabei die Positionsänderung der SPD bei den besonders kontroversen Themen und Abstimmungen in den Analysezeiträumen III und IV. Grundsätzlich lassen sich vor allem Gemeinsamkeiten in den Analysezeiträumen I und II sowie III, IV und V finden, wobei die Parteien in den Zeiträumen II und V eher der allgemeinen ideologischen Wettbewerbslogik folgen und sich in den Zeiträumen I, III und IV anhand der parlamentarischen Wettbewerbslogik positionieren. Die SPD ist im zweiten und im letzten Zeitfenster in der ideologischen Mitte der Debatte bzw. im zweiten Zeitraum deutlich in der Nähe der Oppositionsparteien positioniert und tritt in den anderen Zeiträumen an ihre Koalitionspartner heran. Dies lässt sich durch den hohen Problemdruck, d.h. die hohen Asylantragszahlen in Deutschland in den Analysezeiträumen III und IV, und durch die Verhandlungen und Abstimmungen zum Asylpaket I und II erläutern, die zur erfolgreichen Aushandlung und parlamentarischen Umsetzung einer hohen Koalitionsgeschlossenheit bedurften. Darüber hinaus lässt sich vermuten, dass die mediale Berichterstattung und die damit einhergehende Beeinflussung der öffentlichen Meinung den Problemdruck und Zugzwang vor allem für die Regierungsparteien erhöhte (Boswell 2009b). Hier greift das von Hollifield (2015) konzeptualisierte Dilemma, das liberale Staaten insbesondere in der Migrationspolitik unter Druck setzt, ihren liberalen Handlungsmaximen zu entsprechen und zugleich dem Ziel gerecht zu werden, der einheimischen Bevölkerung möglichst gute wirtschaftliche Bedingungen zu gewährleisten oder den Zugang zu Sozialsystemen zu regulieren.

Die SPD, die ideologisch gemäßigt links und laut Chapel-Hill-Expert-Survey fast identisch mit Bündnis 90/Die Grünen positioniert ist (Polk et al. 2017), befin- 
det sich mit ihrer Zugehörigkeit zur Mitte-Rechts-Koalition der CDU/CSU-Fraktion damit besonders der Regierungslogik und einem Rechtfertigungszwang ausgesetzt. Als sozialdemokratische Partei muss sie strengere Regulierungen und Zugangsbeschränkungen mit ihren liberalen Grundsätzen vereinbaren. Dies tut sie mit dem Ziel, die Regierungskoalition nicht zu gefährden, da die Koalition ihr strategisch die beste Möglichkeit gibt, aktiv an der Regierungstätigkeit teilzunehmen. Hier zeigt sich nicht nur das liberale Dilemma des Politikfelds Migration, sondern ein Problem der Catch-All-Partei SPD, die mit schwindender Parteienidentifikation und einem Wandel der Konfliktlinien in der deutschen Gesellschaft sowie internen Problemen zu kämpfen hat (Jun 2019). Durch den jahrelangen Kurs einer Großen Koalition fällt es ihr zudem schwer, sich glaubwürdig von der CDU abzugrenzen und eigene programmatische Punkte im Regierungsprogramm zu platzieren.

Darüber hinaus ist es die Multidimensionalität des Politikfeldes Migration, die es den Parteien und insbesondere der SPD erlaubt bzw. sogar zwingt, zu unterschiedlichen Zeitpunkten unterschiedliche Wettbewerbslogiken zu verfolgen (Lutz 2019; Money 1999). Je nach Themenfokus auf Migrationsregulation, Integration oder Flüchtlingspolitik in den Analysezeiträumen, die sich während der Legislaturperiode und der Flüchtlingskrise in Deutschland deutlich unterscheiden, bietet dies für die Parteien Anlass, ihre Positionierung strategisch anzupassen.

Der Regierungs- und Oppositionskonflikt ist der am deutlichsten beobachtbare Einflussfaktor auf die Parteipositionen im Parlament. Die Oppositionsparteien waren im Untersuchungszeitraum sehr geschlossen, weil weder die AfD noch die FDP im Bundestag vertreten waren. Richtig deutlich wird die Regierungs-Oppositionslogik erst anhand des Hin- und Herwechselns der SPD. Um eine produktive und effiziente Regierungszusammenarbeit zu gewährleisten, sind die Parteien der Regierungskoalition gezwungen, gemeinsame Positionen zu vertreten, auch wenn ideologische oder wahlstrategische Erwägungen ggf. ganz andere Positionierungen nahelegen würden. Auch das ist im Falle der Flüchtlingskrise in Deutschland eine interessante Konstellation, da die Aushandlungen zum Koalitionsvertrag, wie beschrieben, sehr konfliktreich verlaufen sind und auch die öffentliche Debatte während der Flüchtlingskrise keine absolute Geschlossenheit der Regierungsparteien vermuten ließ. Dies betrifft nicht nur die SPD, sondern auch das Verhältnis der CDU und CSU, die sich im öffentlichen Diskurs scharf gegenseitig kritisierten (Handelsblatt 2018; Süddeutsche Zeitung 2016a), ohne dass sich dieser Konflikt in die Parlamentsdebatten zu übersetzen scheint. Die Bewegung der SPD im Analysezeitraum V in die Mitte der Dimension könnte dann ebenso mit der schwindenden medialen Aufmerksamkeit (Haller 2017, 2019), sinkenden Asylantragzahlen (Bundesamt für Migration und Flüchtlinge 2018, 2019) und weniger umstrittenen Abstimmungen wie zum neuen Integrationsgesetz (Deutscher Bundestag 2016) zusammenhängen, sodass sie sich entsprechend der allgemeinen Wettbewerbslogik in wachsender Distanz zu den Koalitionsparteien positioniert.

Es bleibt offen, wie liberale Staaten und Regierungsparteien in Zukunft durch die wachsenden Migrationsströme in Zeiten zunehmender Vernetzung und Globalisierung im politikfeldspezifischen Parteienwettbewerb agieren. Nicht nur die aktuelle Eskalation an der türkisch-griechischen Grenze zeigen die anhaltende Relevanz der Thematik für Europa (Süddeutsche Zeitung 2020). Auch in Deutschland wird Flucht 
und Migration als Dimension des Parteienwettbewerbs nicht an Bedeutung verlieren und die etablierten Parteien werden Kompetenzen und Lösungsstrategien anbieten müssen. Vor allem wenn der Druck durch die Etablierung und den anhaltenden Erfolg von rechtspopulistischen Parteien, als Issue Owner im Politikfeld Migration, auf die etablierten Parteien steigt. Mit dem Eintreten der AfD in den Deutschen Bundestag im Jahr 2017 und den Wiedereintritt der FDP entsteht eine komplexe Wettbewerbsdynamik, die die SPD noch mehr unter Druck setzt und das fragile Konstrukt der Großen Koalition als überholtes Erfolgskonzept in Deutschland grundlegend in Frage stellt.

Funding Open Access funding provided by Projekt DEAL.

Open Access Dieser Artikel wird unter der Creative Commons Namensnennung 4.0 International Lizenz veröffentlicht, welche die Nutzung, Vervielfältigung, Bearbeitung, Verbreitung und Wiedergabe in jeglichem Medium und Format erlaubt, sofern Sie den/die ursprünglichen Autor(en) und die Quelle ordnungsgemäß nennen, einen Link zur Creative Commons Lizenz beifügen und angeben, ob Änderungen vorgenommen wurden.

Die in diesem Artikel enthaltenen Bilder und sonstiges Drittmaterial unterliegen ebenfalls der genannten Creative Commons Lizenz, sofern sich aus der Abbildungslegende nichts anderes ergibt. Sofern das betreffende Material nicht unter der genannten Creative Commons Lizenz steht und die betreffende Handlung nicht nach gesetzlichen Vorschriften erlaubt ist, ist für die oben aufgeführten Weiterverwendungen des Materials die Einwilligung des jeweiligen Rechteinhabers einzuholen.

Weitere Details zur Lizenz entnehmen Sie bitte der Lizenzinformation auf http://creativecommons.org/ licenses/by/4.0/deed.de.

Interessenkonflikt D.S. Atzpodien gibt an, dass kein Interessenkonflikt besteht.

\section{Literatur}

Aydogan, Abdullah, und Jonathan B. Slapin. 2015. Left-right reversed. Party Politics 21:615-625.

Bale, Tim. 2003. Cinderella and her ugly sisters: the mainstream and extreme right in Europe's bipolarising party systems. West European Politics 26:67-90.

Benoit, Kenneth, Kohei Watanabe, Haiyan Wang, Paul Nulty, Adam Obeng, Stefan Müller, und Akitaka Matsuo. 2018. quanteda: An R package for the quantitative analysis of textual data. Journal of Open Source Software 3:774.

Bieber, Christoph, Andreas Blätte, Karl-Rudolf Korte, und Niko Switek (Hrsg.). 2017. Regieren in der Einwanderungsgesellschaft. Wiesbaden: Springer.

Blätte, Andreas. 2015. Migrationspolitik in der Vergleichenden Politikwissenschaft. In Handbuch Vergleichende Politikwissenschaft, Hrsg. Hans-Joachim Lauth, Marianne Kneuer, und Gert Pickel, 767-781. Wiesbaden: Springer.

Borkert, Maren, und Wolfgang Bosswick. 2011. The case of Germany. In Migration policymaking in Europe. The dynamics of actors and contexts in past and present, Hrsg. Giovanna Zincone, Maren Borkert, und Rinus Penninx, 95-128. Amsterdam: Amsterdam University Press.

Boswell, Christina (Hrsg.). 2009a. The political uses of expert knowledge. Immigration policy and social research. Cambridge: Cambridge University Press.

Boswell, Christina. 2009b. The politics of immigration in Germany and the UK. In The political uses of expert knowledge. Immigration policy and social research, Hrsg. Christina Boswell, 107-129. Cambridge: Cambridge University Press.

Brettell, Caroline, und James F. Hollifield (Hrsg.). 2008. Migration theory. Talking across disciplines, 2. Aufl., New York: Routledge.

Budge, Ian. 1994. A new spatial theory of party competition: uncertainty, ideology and policy equilibria viewed comparatively and temporally. British Journal of Political Science 24:443.

Bundesamt für Migration und Flüchtlinge. 2018. Das Bundesamt in Zahlen 2017. Asyl, Integration und Migration 
Bundesamt für Migration und Flüchtlinge. 2019. Aktuelle Zahlen: Ausgabe Juni 2019. Tabellen, Diagramme, Erläuterungen

Bundestag. 2016. Bundestag beschließt ein Integrationsgesetz. https://www.bundestag.de/dokumente/ textarchiv/2016/kw27-de-integrationsgesetz-433728. Zugegriffen: 23. Okt. 2019.

Bundesverfassungsgericht. 2016. Zum Urteil des Zweiten Senats vom 3. Mai 2016. http://www.bverfg.de/ e/es20160503_2bve000414.html. BVerfGE 142, 25-74. Zugegriffen: 17. Mai 2019.

Calhoun, Craig J. 2007. Nations matter. Culture, history and the cosmopolitan dream. London: Routledge.

Catalinac, Amy. 2018. Positioning under alternative electoral systems: evidence from Japanese candidate election manifestos. American Political Science Review 112:31-48.

Dahlström, Carl, und Peter Esaiasson. 2013. The immigration issue and anti-immigrant party success in Sweden 1970-2006. Party Politics 19:343-364.

Deutscher Bundestag. 15.10.2015a. Namentliche Abstimmung. Gesetzentwurf der Fraktionen der CDU/CSU und SPD Entwurf eines Asylverfahrensbeschleunigungsgesetzes, hier: Artikel 1 Nummer 35 des Gesetzentwurfs in der Ausschussfassung (Neufassung der Anlage II zu § 29a des Asylverfahrensgesetzes) (Drucksachen 18/6185 und 18/6386). https://www.bundestag.de/parlament/plenum/ abstimmung/abstimmung?id=366. Zugegriffen: 11. Juli 2018.

Deutscher Bundestag. 15.10.2015b. Namentliche Abstimmung. Gesetzentwurf der Fraktionen der CDU/CSU und SPD Entwurf eines Asylverfahrensbeschleunigungsgesetzes, hier: Artikel 2 des Gesetzentwurfs in der Ausschussfassung (Änderung des Asylbewerberleistungsgesetzes) (Drucksachen 18/6185 und 18/6386). https://www.bundestag.de/parlament/plenum/abstimmung/abstimmung? id=362. Zugegriffen: 11. Juli 2018.

Deutschlandradio. 2013. Große Koalition ist für SPD-Basis ,ganz schwierig“. Deutschlandfunk Kultur. 26. Sept. 2013.

Dolezal, Martin, Laurenz Ennser-Jedenastik, Wolfgang C. Müller, und Anna K. Winkler. 2016. Analyzing manifestos in their electoral context a new approach applied to Austria, 2002-2008. Political Science Research and Methods 4:641-650.

Dostal, Jörg M. 2017a. The crisis of German social democracy revisited. The Political Quarterly $88: 230-240$.

Dostal, Jörg M. 2017b. The German federal election of 2017: how the wedge issue of refugees and migration took the shine off chancellor Merkel and transformed the party system. The Political Quarterly 88:589-602.

FAZ (Hrsg.). 2015. Behörden überlastet. 300.000 Flüchtlinge ohne Asylantrag in Deutschland. 26.11.2015. https://www.faz.net/aktuell/politik/fluechtlingskrise/300-000-fluechtlinge-ohne-asylantrag-indeutschland-13933328.html. Zugegriffen: 11. März 2020.

FAZ. 2013. Sieben Juso-Landesverbände gegen große Koalition. FAZ. 2. Dez. 2013. Gesehen 17. Mai 2019.

Franzmann, Simon T. 2016. Opposition und Staat. Zur Grundlegung der Parteiendemokratie. In Parteien in Staat und Gesellschaft, Hrsg. Sebastian Bukow, Uwe Jun, und Oskar Niedermayer, 51-83. Wiesbaden: Springer.

Frid-Nielsen, Snorre S. 2018. Human rights or security? Positions on asylum in European Parliament speeches. European Union Politics 19:344-362. https://doi.org/10.1177/1465116518755954.

Gestmann, Margarita, und Markus Hilz. 2017. Das Politikfeld Migrations- und Integrationspolitik. In Implementation in Politikfeldern, Hrsg. Dieter Grunow, 217-285. Wiesbaden: Springer.

Givens, Terri, und Adam Luedtke. 2004. The politics of European Union immigration policy: institutions, salience, and harmonization. Policy Studies Journal 32:145-165.

Givens, Terri, und Adam Luedtke. 2005. European immigration policies in comparative perspective: issue salience, partisanship and immigrant rights. Comparative European Politics 3:1-22.

Green, Simon. 2006. Zwischen Kontinuität und Wandel: Migrations- und Staatsangehörigkeitspolitik. In Regieren in der Bundesrepublik Deutschland, Hrsg. Manfred G. Schmidt, Reimut Zohlnhöfer, 113-134. Wiesbaden: VS.

Green-Pedersen, Christoffer, und Jesper Krogstrup. 2008. Immigration as a political issue in Denmark and Sweden. European Journal of Political Research 47:610-634.

Grimmer, Justin, und Brandon M. Stewart. 2013. Text as data. The promise and pitfalls of automatic content analysis methods for political texts. Political Analysis 21:267-297.

Haller, Michael. 2017. Die „Flüchtlingskrise“ in den Medien. Tagesaktueller Journalismus zwischen Meinung und Information. Frankfurt am Main: Otto Brenner Stiftung.

Haller, Michael. 2019. Zwischen „Flüchtlingskrise“ und „,Migrationspakt“. Mediale Lernprozesse auf dem Prüfstand. Frankfurt am Main: Otto Brenner Stiftung. 
Handelsblatt. 2018. Merkel, Seehofer und die Flüchtlingspolitik - So eskalierte der Asylstreit. https://www. handelsblatt.com/politik/deutschland/chronologie-merkel-seehofer-und-die-fluechtlingspolitik-soeskalierte-der-asylstreit/22756616.html?ticket=ST-7593024-i27lkR9A1kmLQfjNg51G-ap1 (Erstellt: 1. Juli 2018). Zugegriffen: 11. März 2020.

Heckmann, Friedrich, und Delia Wiest. 2015. Research-policy dialogues in Germany. In Integrating immigrants in Europe, Bd. 5, Hrsg. Peter Scholten, et al., 185-211. Cham: Springer.

Hess, Christin, und Simon Green. 2016. Introduction: the changing politics and policies of migration in Germany. German Politics 25:315-328.

Hohl, Karina. 2017. Heikles Thema, neue Strategien? In Regieren in der Einwanderungsgesellschaft, Hrsg. Christoph Bieber, et al., 41-46. Wiesbaden: Springer.

Hollifield, James F. 2015. Debunking American exceptionalism and rescuing liberalism. Ethnic and Racial Studies 38:1312-1318.

Jakobs, Simon, und Uwe Jun. 2018. Parteienwettbewerb und Koalitionsbildung in Deutschland 2017/18: Eine Analyse der Wahlprogramme. Zeitschrift für Parlamentsfragen 49:265-285.

Jun, Uwe. 2019. Die SPD in der Ära Merkel: Eine Partei auf der Suche nach sich selbst. In Zwischen Stillstand, Politikwandel und Krisenmanagement, Hrsg. Reimut Zohlnhöfer, Thomas Saalfeld, 39-62. Wiesbaden: Springer.

Klüver, Heike. 2012. Die Macht der Informationen: Eine empirische Analyse von Lobbyingerfolg in der Europäischen Union. Politische Vierteljahresschrift 53:211-239.

Kortmann, Matthias, und Christian Stecker. 2017. Party competition and immigration and integration policies. A comparative analysis. Comparative European Politics 42:2087.

Kriesi, Hanspeter, Edgar Grande, Romain Lachat, Martin Dolezal, Simon Bornschier, und Timotheos Frey. 2006. Globalization and the transformation of the national political space: Six European countries compared. European Journal of Political Research 45:921-956.

Lo, James, Sven-Oliver Proksch, und Jonathan B. Slapin. 2016. Ideological clarity in multiparty competition. A new measure and test using election manifestos. British Journal of Political Science 46:591-610.

Lutz, Philipp. 2019. Variation in policy success: radical right populism and migration policy. West European Politics 42:517-544.

Marcinkiewicz, Kamil, und Markus Tepe. 2012. Positionen, Fraktionen und Mandate. Eine Anwendung der quantitativen Textanalyse auf die Kurzbiographien der Abgeordneten des 17. Deutschen Bundestages $=$ Positions, factions and mandates; applying quantitative text analysis to self-reported biographical notes from the members of the $17^{\text {th }}$ German Bundestag. Methoden, Daten, Analysen: mda : Zeitschrift für empirische Sozialforschung 6:99-132. https://doi.org/10.12758/mda.2012.007.

Mayring, Philipp. 2015. Qualitative Inhaltsanalyse. Grundlagen und Techniken, 12. Aufl., Weinheim: Beltz.

Merkel, Wolfgang. 2017. Kosmopolitismus versus Kommunitarismus: Ein neuer Konflikt in der Demokratie. In Parties, governments and elites, Hrsg. Philipp Harfst, Ina Kubbe, und Thomas Poguntke, 9-23. Wiesbaden: Springer.

Miles, Patrick. 2009. Snowball: A language for stemming algorithms. http://snowballstem.org/credits.html. Zugegriffen: 16. Juli 2018. German.

Money, Jeanette. 1999. Defining immigration policy: Inventory, quantitative referents, and empirical regularities. Annual meeting of the American Political Science Association, Atlanta.

NDR. 2016. Wie die Übergriffe die Flüchtlingsdebatte verändern. https://www.ndr.de/fernsehen/sendungen/ panorama3/Wie-die-Uebergriffe-die-Fluechtlingsdebatte-veraendern, silvesternacht128.html (Erstellt: 12. Jan. 2016). Zugegriffen: 11. März 2020.

Niedermayer, Oskar. 2015. Eine neue Konkurrentin im Parteiensystem? Die Alternative für Deutschland. In Die Parteien nach der Bundestagswahl 2013, Hrsg. Oskar Niedermayer, 175-207. Wiesbaden: Springer.

Niedermayer, Oskar. 2018. Die Entwicklung des bundesdeutschen Parteiensystems. In Handbuch der deutschen Parteien, Hrsg. Frank Decker, Viola Neu, 97-125. Wiesbaden: Springer.

Odmalm, Pontus. 2011. Political Parties and 'the Immigration Issue': Issue Ownership in Swedish Parliamentary Elections 1991-2010. West European Politics 34:1070-1091.

Petrocik, John R. 1996. Issue ownership in presidential elections, with a 1980 case study. American Journal of Political Science 40:825-850. https://www.jstor.org/stable/2111797.

Polk, Jonathan, Jan Rovny, Ryan Bakker, Erica Edwards, Liesbet Hooghe, Seth Jolly, Jelle Koedam, Filip Kostelka, Gary Marks, Gijs Schumacher, Marco Steenbergen, Milada Vachudova, und Marko Zilovic. 2017. Explaining the salience of anti-elitism and reducing political corruption for political parties in Europe with the 2014 Chapel Hill Expert Survey data. Research \& Politics 4:205316801668691. 
Porter, Martin, und Richard Bolton. 2001. Snowball: a language for stemming algorithms. http:// snowballstem.org/. Zugegriffen: 17. Mai 2019.

Proksch, Sven-Oliver, und Jonathan B. Slapin. 2010. Position taking in European parliament speeches. British Journal of Political Science 40:587-611.

Riker, William H. 1993. Agenda formation. Ann Arbor: Univ. of Michigan Press.

Rosenblum, Marc R., und Wayne A. Cornelius. 2012. Dimensions of immigration policy. In Oxford handbook of the politics of international migration, Hrsg. Marc R. Rosenblum, Daniel J. Tichenor. Oxford: Oxford University Press. https://doi.org/10.1093/oxfordhb/9780195337228.013.0011.

Slapin, Jonathan B., und Sven-Oliver Proksch. 2008. A scaling model for estimating time-series party positions from texts. American Journal of Political Science 52:705-722.

Spiegel Online. 2013. Jusos stimmen gegen Große Koalition. Kongress in Nürnberg. https://www.spiegel. de/politik/deutschland/jusos-stimmen-mehrheitlich-gegen-grosse-koalition-a-937802.html (Erstellt: 7. Dez. 2013). Zugegriffen: 17. Mai 2019.

Spiegel Online. 2015a. Flüchtlingskrise überfordert deutsche Behörden. https://www.spiegel.de/politik/ deutschland/fluechtlinge-in-deutschland-warum-so-viel-chaos-und-leid-a-1046851.html (Erstellt: 11. Aug. 2015). Zugegriffen: 11. März 2020.

Spiegel Online. 2015b. Uneinige Koalition: Asylverschärfung verzögert sich. Flüchtlingskrise: CDU CSU SPD streiten über Asylpaket. http://www.spiegel.de/politik/deutschland/fluechtlingskrise-cdu-csuspd-streiten-ueber-asylpaket-a-1065337.html (Erstellt: 30. Nov. 2015). Zugegriffen: 21. Jan. 2019.

Süddeutsche Zeitung. 2013. Große Koalition, großes Risiko. https://www.sueddeutsche.de/politik/ moeglicher-mitgliederentscheid-der-spd-grosse-koalition-grosses-risiko-1.1780898 (Erstellt: 26. Sept. 2013). Zugegriffen: 17. Mai 2019.

Süddeutsche Zeitung. 2016a. Flüchtlingskrise: CSU widerspricht der CSU. https://www.sueddeutsche.de/ politik/fluechtlingskrise-csu-widerspricht-der-csu-1.2915342 (Erstellt: 20. März 2016). Zugegriffen: 14. Jan. 2019.

Süddeutsche Zeitung. 2016b. Jeder kämpft für sich allein. https://www.sueddeutsche.de/politik/koalitionjeder-kaempft-fuer-sich-allein-1.2938561 (Erstellt: 7. Apr. 2016). Zugegriffen: 14. Jan. 2019.

Süddeutsche Zeitung. 2020. Was wir erleben, ist eine kontrollierte Eskalation. https://www.sueddeutsche. de/politik/fluechtlinge-deutschland-griechenland-1.4832866 (Erstellt: 6. März 2020). Zugegriffen: 11. März 2020.

Wayne, Cornelius, Philip Martin, und James Hollifield (Hrsg.). 1994. Controlling immigration: a global perspective. Stanford: Stanford University Press.

welt.de. 2015. Behörden überlastet. Flüchtlingskrise deckt Schwächen des deutschen Staates auf. https://www.welt.de/politik/deutschland/article146598658/Fluechtlingskrise-deckt-Schwaechendes-deutschen-Staates-auf.html (Erstellt: 20. Sept. 2015). Zugegriffen: 11. März 2020.

Wiesendahl, Elmar. 2016. Der Kulturkonflikt um die Flüchtlingskrise und die politischen Folgen. Zeitschrift für Staats- und Europawissenschaften 14:53-79. 